\title{
Numerical simulation of a high viscosity bubble column
}

\author{
$\underline{\text { Danilo Carvajal }}^{\mathrm{a}}$, Victor Melendez-Vejar ${ }^{\mathrm{a}}$, Maik Irrázabal $^{\mathrm{a}}$ and Carlos Carlesi-Jara ${ }^{\mathrm{a}}$ \\ ${ }^{a}$ Pontificia Universidad Católica de Valparaíso, Escuela de Ingeniería Química, Av. Brasil 2950, \\ Valparaíso 2340025, Chile \\ Email:danilo.carvajal@ucv.cl.
}

\begin{abstract}
The objective of this work is to develop fluid dynamic model to simulate a high viscosity bubble column for $\mathrm{CO}_{2}$ absorption in Ionic Liquids (ILs). A very promising solvent for $\mathrm{CO}_{2}$ capture and conversion are ionic liquids (ILs); ILs consist of a wide group of salts, which are liquids at room temperature, have low vapor pressure, high ionic conductivity and thermal stability. However, the use of ILs for industrial $\mathrm{CO}_{2}$ depletion has a series of technical and economic issues that must be solved if this strategy is to be implemented. A very important drawback of ILs used for gas removal is its high viscosity, reaching values above $0.010 \mathrm{~Pa} \cdot \mathrm{s}$ which results in a decrease of the overall mass transfer rate and ancrease in the power required for pumping and mixing. In order to elucidate the hydrodynamic behavior in a bubble column for $\mathrm{CO}_{2}$ absorption with one gas feed inlet, a Computational Fluid Dynamic (CFD) model was developed, which was experimentally validated through a laboratory scale bubble column. To simplify the calculations and increase the accuracy of the results, the system was modeled as a single rising bubble which permits the estimation of the bubble rising velocity and the change of the bubble shape and size during its displacement. The model approach consists in a simplified two-dimensional multiphase flow model which considers the liquid solvent as a Newtonian fluid. The laminar, isothermal, and non-stationary hypotheses for both phases is applied. To model the displacement of the gas-liquid interface, the Level Set method was used. The laboratory tests were carried out using water-glycerol mixtures $(58 \%, 78 \%, 84 \%$ and $88 \%$ by weight) and two Imidazolium type ionic liquids (pure $[\mathrm{bmim}] \mathrm{BF}_{4}$ and $[\mathrm{bmim}] \mathrm{PF}_{6}$ ). To compare the results obtained from the laboratory and the simulations, the drag coefficient for gas bubbles in liquids was used which correlates the fluid physical properties of fluids and the bubble equivalent diameter and terminal velocity. The results were also compared with predicted values obtained through a new correlation for the drag coefficient of single rising bubbles in ILs proposed by Dong et al. (2010). The results indicated that the CFD model is in good agreement with the experimental results, particularly for bubble Reynolds numbers below 5. Above this value, the model tends to underestimate the bubble terminal velocity which can be explained by the effect of the high velocity gradients close to the gas-liquid interface. Future steps will involve improving of the computational mesh, a parametric analysis of the reintialization parameter and the parameter controlling the thickness at the interface transition zone. Acknowledgments. This work was supported by FONDECYT postdoc $\mathrm{N}^{\circ} 3120138$ from CONICYT (Chile).
\end{abstract}

Keywords: Computational Fluid Dynamic, Bubble Column, Ionic Liquid. 


\section{INTRODUCTION}

The continuous increase in $\mathrm{CO}_{2}$ atmospheric concentration has caused a great concern in the scientific community because of its environmental hazard and its social implications. The European Union has set a target of $20 \%$ reduction of $\mathrm{CO}_{2}$ emission by the year 2020; to meet this goal, a significant reduction in the $\mathrm{CO}_{2}$ releases from fossil fuel will be required during the next years. This can be achieved by adopting an effective strategy for carbon capture, such as the use of liquids solvents for $\mathrm{CO}_{2}$ capture. The ionic liquids present several characteristics which make them very interesting for $\mathrm{CO}_{2}$ capture applications. However, when a gas is absorbed into an ionic liquid, typically the viscosity suffers an increase which has several negative impacts including the reduction of the species transport rate across the solvent and the increase the energy required for pumping and mixing. The physical properties of ILs may be modified by changing the substitute group in the cations and anions (Galán Sánchez et al., 2007). During the gas absorption process, the increasing viscosity process may be controlled by a continuous removal of the solvent before the viscosity increases in a decontrolled way. There are very few theoretical and experimental investigations focusing on bubble columns using ILs as solvent one being the work by Dong et al. (2010), who conducted experiments using the ionic liquids $[\mathrm{bmim}] \mathrm{BF}_{4},[\mathrm{omim}] \mathrm{BF}_{4}$ and $[\mathrm{bmim}] \mathrm{PF}_{6}$ at different operating conditions (i.e. temperature, gas flow rate and gas inlet diameter) in a bubble column fed with pure nitrogen. From the experimental results, they proposed a new correlation for the estimation of the drag coefficient as a function of Reynolds number and bubble aspect ratio as a function of a new dimensionless parameter; both correlations are particularly applicable to gas bubble formation in ionic liquids and demonstrated to be in good agreement with experimental results. Dong et al. (2010) then developed a coupled Computational Fluid Dynamic and Population Balance Model to study the mass transfer during $\mathrm{CO}_{2}$ absorption in ILs (Wang et al., 2010). However, there has been not undertaking as yet into investigation of bubble columns with ILs focused at the micro-scale level. An in-depth knowledge of the interface shape and displacement is important particularly of the mass transfer at a local scale.

\section{EXPERIMENTAL SECTION}

\subsection{Test fluids and physical properties}

Four water-glycerol mixtures $(58 \%, 78 \%, 84 \%$ and $88 \%$ by weight) were prepared by stirring distilled water with the appropriate mass of glycerol for $60 \mathrm{~min}$. Ionic liquids [bmim] $\mathrm{BF}_{4}$ and $[\mathrm{bmim}] \mathrm{PF}_{6} \mathrm{were}$ purchased from Iolitec GmbH (Denzlingen, Germany) with a purity of above $98 \%$. All the fluids were analyzed to determine density, viscosity and surface tension. All the measurements were carried out at $20^{\circ} \mathrm{C}$ to match the conditions of laboratory bubble column procedures. The density was measured using a pycnometer, the viscosity was obtained with an Ostwald viscometer (and compared with a rotational Fungilab viscometer) and the surface tension was measured with a stalagmometer. The physical properties of the tested fluids are listed in Table 1.

Table 1. Physical properties of the test fluids

\begin{tabular}{|c|c|c|c|c|}
\hline Fluid & Description & Density, $\mathbf{~ k g} / \mathbf{m}^{\mathbf{3}}$ & Viscosity, Pa.s & Surface tension, N/m \\
\hline A1 & Glycerol 58 \% & 1155.2 & 0.0097000 & 0.0545711 \\
\hline A2 & Glycerol 78 \% & 1211.8 & 0.0485300 & 0.0484380 \\
\hline A3 & Glycerol 84 \% & 1224.5 & 0.1112900 & 0.0477220 \\
\hline A4 & Glycerol 88 \% & 1282.2 & 0.2358300 & 0.0475000 \\
\hline A5 & BmimBF4 & 1039.3 & 0.1117900 & 0.0289316 \\
\hline A6 & BmimPF6 & 1276.4 & 0.3366855 & 0.0331631 \\
\hline
\end{tabular}

\subsection{Experimental setup}

A schematic diagram of the experimental setup is depicted in Figure 1. The bubble column was made of transparent acrylic (Fig. 2) with a square base of $5 \mathrm{~cm}$ each side and $12 \mathrm{~cm}$ height (total volume of $300 \mathrm{ml}$ ). The column was tightly sealed to prevent leakage of both liquid and gas phases, and to avoid contamination with ambient air. The column has a thermal jacket (fluid: water) to maintain the temperature fixed at $20{ }^{\circ} \mathrm{C}$. Pure nitrogen gas is injected through a single orifice in the bottom of the column. The gas flow rate is controlled by a mass flow controller, and, the gas flow rate constantly checked with a soap film meter. To produce different sizes of bubbles for each fluid tested; two diameters of orifice $(0.8 \mathrm{~mm}$ and $1.25 \mathrm{~mm})$ and two gas flow rates $(7 \mathrm{ml} / \mathrm{min}$ and $20 \mathrm{ml} / \mathrm{min})$ were applied. The size and shape of the bubbles were recorded with a digital camcorder (Panasonic HC V500M) set with a resolution of 1080 x 1920 pixels, 59.94 frames 
per second (progressive) and 1/2000 s of shooter speed. To properly illuminate the column and avoid heating the column during the tests; a $30 \mathrm{~W}$ led lamp (equivalent to a $200 \mathrm{~W}$ halogen lamp) was used. To obtain good image quality (brightness and contrast) a semitransparent (white) acrylic panel was placed between the column and the video camera, which acted as a light diffusion filter. The image analysis was carried out through a MATLAB script developed by our workgroup. The script determine of the position and shape of the bubble in each frame of the recorded video file.

\subsection{Testing procedure}

Before starting the testing procedure, the column is washed and dried to eliminate all residues from previous tests, and then the testing fluid is injected inside the column from the top side until reaching a liquid level of $60 \mathrm{~mm}$ above the gas inlet orifice. The liquid is slowly injected to avoid the formation of bubbles which may hinder the recording process. By controlling the gas flow rate and stabilizing the frequency of formation of bubbles, the bubble motion is then recorded during 30 s to obtain a wide sample of bubbles to analyse. Before and after each test, measurements of temperature and gas flow rate are performed.

\section{CFD MODEL}

\subsection{Basic equations}

The general assumptions for the model used are the laminar, incompressible and isothermal flows. The governing continuity and momentum equations for the two-phase flow are as follows:

$\nabla \cdot \mathbf{u}=\mathbf{0}$,

$\rho \frac{\partial \mathbf{u}}{\partial \mathbf{t}}+\mathbf{u} \cdot \nabla \mathbf{u}=-\frac{1}{\rho} \nabla \mathbf{p}+\frac{\mu}{\rho} \nabla^{2} \mathbf{u}+\mathbf{F}_{\mathbf{v}}$,

respectively, where $\boldsymbol{\rho}$ is the fluid density $\left(\mathrm{kg} / \mathrm{m}^{3}\right), \boldsymbol{\mu}$ the dynamic viscosity $(\mathrm{Pa} \cdot \mathrm{s}), \mathbf{u}$ is the velocity vector $(\mathrm{m} / \mathrm{s}), \mathbf{t}$ is time (s) and $\mathbf{p}$ the total pressure $(\mathrm{Pa})$. The source term $\mathbf{F}_{\mathbf{v}}\left(\mathrm{kg} / \mathrm{m}^{2} \mathrm{~s}\right)$, includes the volumetric forces, such as the gravity force applied to the fluid over the whole domain and the surface tension to the gas-liquid interface.

\subsection{Interface tracking model}

To model the displacement of the gas-liquid interface, the Level Set method was used, which was introduced by Osher and Sethian (1988), for incompressible two phase flow. The motion of the interface is characterized through the scalar function $\phi$, which is a smoothing function where $\phi=0.5$ defines the position of the interface (COMSOL, 2012). For our model, $\phi=0$ defines the gas phase, meanwhile $\phi=1$ defines the liquid phase. The advection equation which defines the transport and reinitialization of $\Phi$ through the entire domain is defined as follows:

$$
\frac{\partial \phi}{\partial \mathbf{t}}+\mathbf{u} \cdot \nabla \phi=\gamma \nabla \cdot\left(\varepsilon \nabla \phi-\phi(1-\phi) \frac{\nabla \phi}{|\nabla \phi|}\right),
$$

where the velocity vector (u) is obtained from the numerical solution of the Navier-Stokes equations (equations 1 and 2). The reintialization parameter $\gamma$ determines the thickness of the interface transition zone 
where $0<\phi<1$. When stabilization techniques for the level set equation are used, it is recommended using a value of $\boldsymbol{\varepsilon}=\mathbf{h} / 2$, where $\mathbf{h}$ is the typical size $(\mathrm{m})$ of the mesh elements in the vicinity of the interface (Osher and Sethian, 1988). The $\gamma$ parameter can be initially approximated by the maximum velocity magnitude of the system. The physical properties such as viscosity and density are estimated from a heaviside function which uses the properties of pure phases:

$$
\rho=\rho_{1}+\left(\rho_{g}-\rho_{1}\right) \cdot \phi
$$

for the density and similarly for the viscosity:

$\mu=\mu_{1}+\left(\mu_{\mathrm{g}}-\mu_{1}\right) \cdot \phi$,

where $\boldsymbol{\rho}_{\mathrm{g}}, \boldsymbol{\rho}_{\mathrm{l}}, \boldsymbol{\mu}_{\mathrm{g}}$ and $\boldsymbol{\mu}_{\mathrm{l}}$, are the density of the gas and the liquid, and viscosities of the gas and the liquid respectively. For the level set method, the surface tension and the gravity force are incorporated into the Navier-Stokes equations as volume forces in the source term of the momentum conservation equation (Eq. 2), which are defined as follows:

$$
\mathbf{F}_{\mathbf{v}}=\boldsymbol{\rho} \cdot \mathbf{g}+\nabla\left[\boldsymbol{\sigma}\left\{\mathbf{I}+\left(-\mathbf{n} \cdot \mathbf{n}^{\mathbf{T}}\right)\right\} \boldsymbol{\delta}\right]
$$

where the first term on the right side defines the gravity force and the second the surface tension, $\sigma$ is the surface tension $(\mathrm{N} / \mathrm{m})$, $\mathbf{I}$ is the identity matrix and $\mathbf{n}$ is a unitary normal vector to the interface with direction from the disperse phase to the continuous phase and is defined as follows (Deshpande, 2006):

$$
\mathbf{n}=\frac{\nabla \phi}{|\nabla \phi|}
$$

The level set parameter $\phi$, is used to approximate the delta function $\boldsymbol{\delta}$ through the following expression:

$$
\delta=6 \cdot|\phi(1-\phi)| \cdot|\nabla \phi|
$$

\subsection{Drag coefficient}

In order to validate the results obtained from the CFD predictions, the drag coefficient obtained from the data produced during the laboratory tests are used. To compare de results with the literature, the drag coefficient correlation proposed by Dong et al. (2010) for bubbly flow using ionic liquids was used. The correlation was developed from experimental data using $[\mathrm{bmim}] \mathrm{BF}_{4},[\mathrm{omim}] \mathrm{BF}_{4}$ and $[\mathrm{bmim}] \mathrm{PF}_{6}$ at different temperatures $\left(37-82^{\circ} \mathrm{C}\right.$, gas flow rate $(0.1-5 \mathrm{ml} / \mathrm{min})$ and gas inlet diameter $(0.17,0.47,0.8$ and $1.4 \mathrm{~mm})$. The empirical expression for the drag coefficient $\left(\mathbf{C}_{\mathbf{d}}\right)$ is based on two dimensionless numbers (i.e. Reynolds and Morton) and is defined as follows (Dong et al., 2010):

$$
\mathbf{C}_{\mathbf{d}}=\mathbf{a} \cdot \mathbf{R e}^{\mathbf{b}} \cdot \mathbf{M o}^{\mathrm{c}}
$$

where $\mathbf{a}, \mathbf{b}$ and $\mathbf{c}$ are defined as following:

For $0.5 \leq \boldsymbol{R e} \leq 5: \mathbf{a}=22.73, \mathbf{b}=-0.849, \mathbf{c}=0.020$, and for $5<\boldsymbol{R e} \leq 50: \mathbf{a}=20.08, \mathbf{b}=-0.636, \mathbf{c}=0.046$. The use of these values will be termed Cd H.Dong (Table 2).

The drag coefficient for spherical bubbles is defined as following:

$\mathbf{C}_{\mathbf{d}}=\frac{4 \cdot \mathbf{d}_{\mathbf{e}} \cdot\left(\rho_{\mathbf{l}}-\rho_{\mathrm{g}}\right) \cdot \mathbf{g}}{3 \cdot \rho_{\mathbf{l}} \cdot \mathbf{v}_{\mathbf{t}}{ }^{2}}$,

where $\mathbf{d}_{\mathbf{e}}$ is the bubble equivalent diameter $(\mathrm{m})$, and $\mathbf{v}_{\mathbf{t}}$ is the terminal rising velocity of bubble $(\mathrm{m} / \mathrm{s})$. Terminal velocity is reached when there is a balance between buoyancy and drag forces. The Reynolds number defined for a single rising bubble and Morton number are defined as:

$$
\begin{aligned}
& \operatorname{Re}=\frac{\rho_{\mathbf{l}} \cdot \mathbf{v}_{\mathbf{t}} \cdot \mathbf{d}_{\mathbf{e}}}{\mu_{\mathrm{l}}}, \\
& \mathbf{M o}=\frac{\mathrm{g} \cdot \mu_{\mathrm{l}}{ }^{4} \cdot\left(\rho_{\mathrm{l}}-\rho_{\mathrm{g}}\right)}{\sigma^{3} \cdot \rho_{\mathrm{l}}{ }^{2}},
\end{aligned}
$$


respectively, where $\mathbf{d}_{\mathbf{e}}$ is calculated as follows:

$\mathbf{d}_{\mathrm{e}}=\sqrt[3]{\mathbf{d}_{\mathbf{x}}^{2} \cdot \mathbf{d}_{\mathbf{y}}}$

where $\mathrm{d}_{\mathrm{x}}$ and $\mathrm{d}_{\mathrm{y}}$ are the horizontal and vertical diameters $(\mathrm{m})$ of the bubble, respectively.

\subsection{Geometry and boundary conditions}

The numerical domain has a height of $60 \mathrm{~mm}$ and a width of $40 \mathrm{~mm}$. The size and aspect ratio of the initial condition of the interface gas-liquid is fixed according to the video

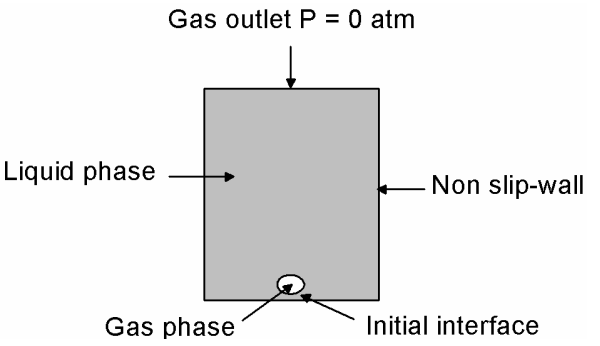

Figure 3. Geometry details and boundary conditions. recording from the experimental procedure (the first frame after the bubble detachment is used). The upper side of the fluid domain is defined as a zero pressure outlet. A non-slip condition is used for the walls. A representation of the geometry and boundary conditions are depicted in Figure 3.

\subsection{Numerical details}

The CFD software COMSOL 4.2 was used for all the simulations. COMSOL uses the finite element method to discretize the partial differential equations defining the mathematical model. Both the geometry and the computational mesh were created in COMSOL. Different two-dimensional grids were tested and a final mesh of 17906 triangular cells was used. The mesh was refined to resolve regions of high gradients efficiently. The final minimum element size was approximately $0.09 \mathrm{~mm}$ in the interface displacement zone. The final element size

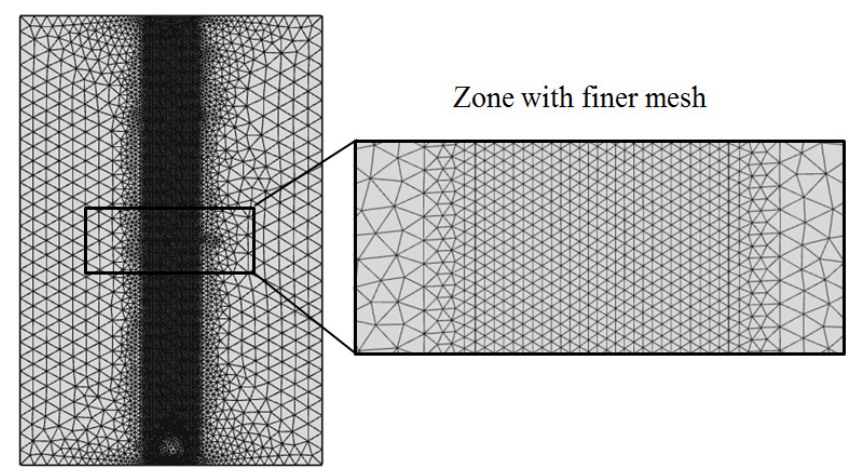

Figure 4. Computational mesh. ranges between 0.09 and $1.43 \mathrm{~mm}$. A representation of the computational mesh is depicted in Figure 4. The system was defined as non-stationary and the total time needed for each simulation was 27.6 hours per second of simulation. The direct PARDISO solver was used for all simulations. Convergence was achieved when all normalized residuals of velocity and concentration reached values smaller than $10^{-3}$. The simulations were performed on a HP Workstation with four 2.26 $\mathrm{GHz}$ Intel cores and 8 GB RAM. In order to ensure stability, the time step was fixed to $0.0001 \mathrm{~s}$. The maximum number of iterations is reached when the relative tolerance exceeds 0.001 with respect of all variables.

\section{RESULTS}

\subsection{Bubble rising velocity}

After detachment, bubbles rise and suffering deceleration reaching a constant terminal velocity when a balance between buoyancy and drag forces is reached. From the experimental data and the simulations it is confirmed that a high viscosity
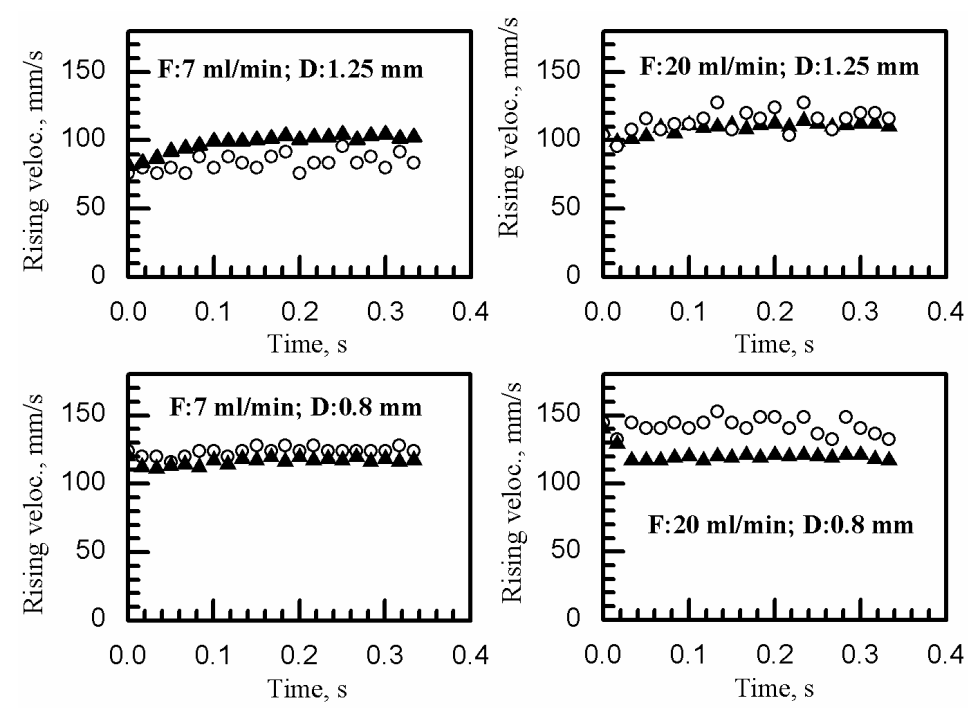

Figure 5. Bubble rising velocity evolution for 4 testing conditions using $[\mathrm{bmim}] \mathrm{BF}_{4}$ as liquid phase $(3.1<\mathrm{Re}<8)$. The triangles represent the CFD calculations and the circles the laboratory experiments. 
leads to a decrease in rising velocity of bubbles. The CFD calculations for the bubble raising velocity presented good agreement with the experimental tests for several applied conditions, particularly for low Reynolds number, below 5. Most experimental fluids had viscosities above 0.1 Pa.s including the glycerol 84 $\%$ and $88 \% \mathrm{w} / \mathrm{w}$, the $[\mathrm{bmim}] \mathrm{BF}_{4}$ and $[\mathrm{bmim}] \mathrm{PF}_{6}$. Experimental and simulated bubble rising velocities for [bmim $] \mathrm{BF}_{4}$ and $\left[\mathrm{bmim} \mathrm{PF}_{6}\right.$ samples are shown in Figure 5 and Figure 6 respectively. The figures show the high accuracy of the simulations for several conditions. However, when the Reynolds number exceeds a value of 5 , the accuracy of the method decreases and the bubble terminal velocities are underestimated. The low accuracy of the CFD calculations at low viscosities can be explained from the effect of the high velocity gradients close to the interface gas-liquid. This problem was partially overcome by performing a finer computational mesh in the vicinity of the interface; however a further improvement of the model is necessary. Improvements would include a parametric analysis of the reintialization parameter and the parameter controlling the thickness at the interface transition zone.

\subsection{Model validation: Drag coefficient}

The Drag coefficients and its associated Reynolds numbers calculated from the laboratory tests and the CFD simulations are graphically depicted in the Figure 7. As can be seen in the graph, experimental results clearly present a power law pattern between $\mathbf{R e}$ and $\mathbf{C d}$. Included in this relationship is the Morton number with which it is possible to estimate the coefficients of equation 9 using the least squares method giving the following parameters: for $\mathbf{R e}$ $\leq 5: \mathbf{a}=58.66, \mathbf{b}=-0.782, \mathbf{c}=$ 0.185 , and for $\operatorname{Re}>5: \quad \mathbf{a}=$ $4.601, \mathbf{b}=-0.605, \mathbf{c}=-0.1471$. The use of these values in equation (9) will be termed $\mathrm{Cd}$ fit (Table 2). These parameters can be used to compare
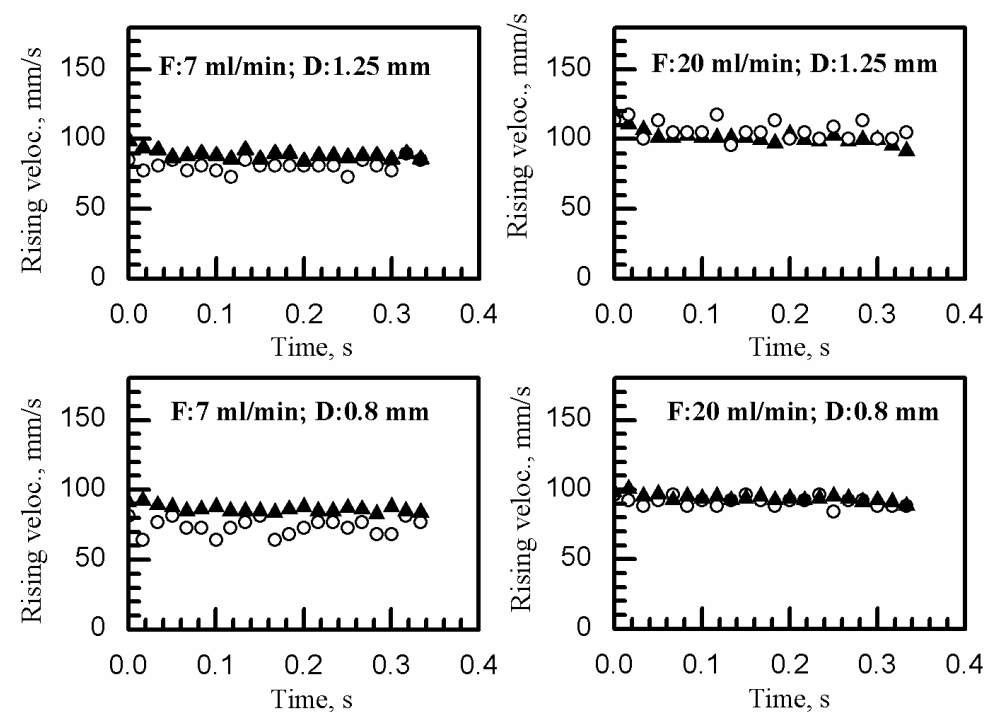

Figure 6. Bubble rising velocity evolution for 4 testing conditions using $[\mathrm{bmim}] \mathrm{PF}_{6}$ as liquid phase $(1.8<\mathrm{Re}<3.0)$. The triangles represent the CFD calculations and the circles the laboratory experiments. experimental results via eqn (9) with CFD simulations. The CFD predictions are consistent with the experimental results for Reynolds numbers below 5. A high deviation between the experiments and CFD predictions were obtained for bubble Reynolds numbers above 20 (Figure 7). The comparison between the Drag coefficients calculated from the CFD model, experimental results using

Table 2. Drag coefficients for the tested ionic liquids with different conditions, using three different approaches: experimental, CFD and H.Dong correlation.

\begin{tabular}{|c|c|c|c|c|c|c|c|}
\hline Fluid & $\mathbf{V}_{\mathrm{t}} \mathbf{m m} / \mathbf{s}$ & $\mathrm{d}_{\mathrm{eq}}, \mathrm{mm}$ & $\mathbf{R e}$ & Mo & $\mathrm{C}_{\mathrm{d}}$ CFD & $C_{d}$ fit experim & $C_{d}$ H.Dong \\
\hline $\operatorname{bmim}\left[\mathrm{BF}_{4}\right]$ & 102.52 & 3.97 & 3.78 & $1.05 \mathrm{E}-03$ & 4.93 & 5.83 & 6.41 \\
\hline $\operatorname{bmim}\left[\mathrm{BF}_{4}\right]$ & 110.48 & 4.63 & 4.76 & $1.05 \mathrm{E}-03$ & 4.96 & 4.87 & 5.27 \\
\hline $\operatorname{bmim}\left[\mathrm{BF}_{4}\right]$ & 116.31 & 5.47 & 5.91 & $1.05 \mathrm{E}-03$ & 5.28 & 4.31 & 4.73 \\
\hline $\operatorname{bmim}\left[\mathrm{BF}_{4}\right]$ & 118.27 & 6.33 & 6.97 & $1.05 \mathrm{E}-03$ & 5.92 & 3.90 & 4.26 \\
\hline $\operatorname{bmim}\left[\mathrm{PF}_{6}\right]$ & 87.99 & 5.94 & 1.98 & $2.59 \mathrm{E}-03$ & 10.02 & 11.41 & 11.30 \\
\hline $\operatorname{bmim}\left[\mathrm{PF}_{6}\right]$ & 100.33 & 7.64 & 2.90 & $2.59 \mathrm{E}-03$ & 9.91 & 8.46 & 8.16 \\
\hline $\operatorname{bmim}\left[\mathrm{PF}_{6}\right]$ & 86.16 & 5.68 & 1.86 & $2.59 \mathrm{E}-03$ & 10.00 & 12.00 & 11.93 \\
\hline $\operatorname{bmim}\left[\mathrm{PF}_{6}\right]$ & 93.90 & 6.63 & 2.36 & $2.59 \mathrm{E}-03$ & 9.83 & 9.94 & 9.73 \\
\hline
\end{tabular}


the equation 9 and the Dong's correlation using equation is shown in Table 2. As can be seen in the table, at very low Reynolds number there is no a clear relationship between the deviation of the CFD results in respect to the experimental results. Given the limited amount of data available for this Reynolds number range, further testing is necessary, including lower gas flow rates and higher viscosity

\section{DISCUSSION AND CONCLUSIONS}

The CFD calculations for the bubble raising velocity presented good agreement in respect to the experimental tests for several applied conditions. In particular, this occurred for instances of Reynolds number below 5, most of experimental fluids had viscosities above 0.1 Pa.s, including, glycerol $84 \%$ and $88 \% \mathrm{w} / \mathrm{w}$, the $\left[\mathrm{bmim}^{\mathrm{B}} \mathrm{BF}_{4}\right.$ and $[\mathrm{bmim}] \mathrm{PF}_{6}$. When the Reynolds number exceeds a value of 5 , the accuracy of the method decreases and the bubble terminal velocities are underestimated. The low accuracy of the CFD calculations at low viscosities can be explained from the effect of the high velocity gradients close to the gas-liquid interface which is related to deformation of the interface, which in turn affects the bubble rising velocity. Future experimentation and modeling will involve improvements in the computational mesh and a parametric analysis of the reintialization parameter and the parameter controlling the thickness at the interface transition zone.

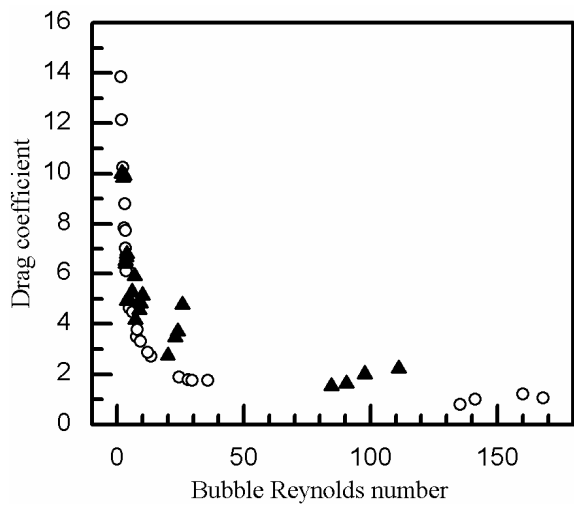

Figure 7. Drag coefficient vs. Reynolds number for several testing conditions (each point represent one test). The triangles represent the CFD calculations and the circles the laboratory experiments.

\section{ACKNOWLEDGMENTS}

This work was supported by FONDECYT N¹111000 project and FONDECYT post-doc N³120138, both from CONICYT (Chile).

\section{REFERENCES}

COMSOL (2012). Product information COMSOL Multiphysics 4.2, Stockholm.

Deshpande, K. B., Zimmerman, W. B. (2006) "Simulation of interfacial mass transfer by droplet dynamics using the level set method. Chemical Engineering Science 61(19): 6486 - 6498.

Dong, H., Wang, X., Liu, L., Zhang, X., Zhang S. (2010). The rise and deformation of a single bubble in ionic liquids. Chemical Engineering Science 65 (10): 3240 - 3248.

Galán Sánchez, L. M., Meindersma, G. W. \& de Haan A.B. (2007). Solvent properties of functionalized ionic liquids for $\mathrm{CO}_{2}$ absorption, Chemical Engineering Research and Design, 85 (1): 31-39.

Osher, S., Sethian J.(1988). Fronts propagating with curvature-dependent speed: Algorithms based on Hamilton-Jacobi formulations, Journal of Computational Physics, 79: 12-49.

Wang, X., Dong, H., Zhang, X., Xu, Y., Zhang, S. (2010). Numerical simulation of absorbing $\mathrm{CO}_{2}$ with ionic liquids. Chemical Engineering Technology, 33(10): 1615-1624. 